\title{
NOTE ON A CLASS OF K-PARANORMAL WEIGHTED COMPOSITION OPERATORS ON THE FOCK SPACE $\mathcal{F}^{p}(\mathbb{C})$
}

\author{
C. SANTHOSHKUMAR AND T. VELUCHAMY
}

\begin{abstract}
In this paper, we characterize the k-paranormal, isometric, spectral radius and the numerical radius of the weighted composition operator $C_{\psi, \phi}$ on the Fock space $\mathcal{F}^{p}(\mathbb{C})$ for $0<p \leq \infty$, where $\psi$ is of the form $\psi(0) e^{-\mu \bar{\nu} \zeta}$ and $\mu, \nu, \zeta \in \mathbb{C}$.
\end{abstract}

\section{INTRODUCTION}

On a space of analytic functions, the composition operator $C_{\phi}$ is deifned as $C_{\phi}(f)=f \circ \phi$ and the weighted composition operator $C_{\psi, \phi}$ is defined as $C_{\psi, \phi}(f)=$ $\psi . f \circ \phi$, where $\phi$ is an analytic self map on $\mathbb{C}$ and $\psi$ is an holomorphic function on $\mathbb{C}$.

The study of composition operators on function spaces attracted various authors over many decades. A vast number of papers appeared on studying the boundedness, compactness, etc. of composition operators acting on function spaces, namely Hardy spaces, Bergmann spaces etc. For more details, refer to $[2,5,8]$.

One of the main objectives of these studies is to find the relationship between the fixed symbol $\phi$ and the weight function $\psi$ with the operator $C_{\psi, \phi}$.

On a space of analytic functions $f$, for $p>0$, the Fock space $\mathcal{F}^{p}(\mathbb{C})$ defined as a space of analytic functions $f$ on $\mathbb{C}$ such that

$$
\|f\|_{p}:=\left(\frac{p}{2 \pi} \int_{\mathbb{C}}|f(\zeta)|^{p} e^{-\frac{p|\zeta|^{2}}{2}} d A(\zeta)^{\frac{1}{p}}<\infty,\right.
$$

where $d A$ is the usual Lebesque measure on $\mathbb{C}$. It is well known that for $1 \leq p \leq \infty$, $\mathcal{F}^{p}(\mathbb{C})$ is a Banach space and for $0<p<1, \mathcal{F}^{p}(\mathbb{C})$ is a complete metric space.

When $p=2, \mathcal{F}^{2}(\mathbb{C})$ is a reproducing kernel Hilbert space (RKHS) with kernel $K_{w}(\zeta)=e^{\zeta \bar{w}}$ and the norm and the inner product are defined respectively as $\|f\|^{2}=\frac{1}{\pi} \int_{\mathbb{C}}|f(\zeta)|^{2} e^{-|\zeta|^{2}} d A(\zeta), \zeta \in \mathbb{C}$ and $\langle f, g\rangle=\frac{1}{\pi} \int_{\mathbb{C}} f(\zeta) \overline{g(\zeta)} e^{-|\zeta|^{2}} d A(\zeta)$, where $d A$ is the usual Lebesque measure on $\mathbb{C}$. In this case, the normalization of $K_{w}$ is denoted as $k_{w}$ (i.e) $k_{w}=\frac{K_{w}}{\left\|K_{w}\right\|}$.

In [1], the authors characterized bounded and compact composition operators on the Fock space $\mathcal{F}^{2}\left(\mathbb{C}^{n}\right)$, the Hilbert space of all holomorphic functions on $\mathbb{C}^{n}$. In [7], the author gave a simple characterization of bounded and compact weighted composition operators on the Fock space $\mathcal{F}^{2}$ over $\mathbb{C}$. On the other hand, in [4],

$M S C$ (2010): primary 47B33, 47A12; secondary $47 \mathrm{~B} 37$.

Keywords: weighted composition operators, k-paranormal, isometric, numerical radius, spectral radius. 
the authors gave criteria for the boundedness and compactness of the weighted composition operators on the Fock space $\mathcal{F}^{p}(\mathbb{C})$. To learn more on the Fock space, one can refer to an excellent book [10]. In [9], the author characterized a special class of unitary weighted composition operators $C_{\psi, \phi}$ and their spectrum on the Fock space $\mathcal{F}^{2}(\mathbb{C})$, where the fixed symbol is of the form $\phi(\zeta)=\mu \zeta-\nu$ and the weighted function is of the form $\psi(\zeta)=\alpha k_{\bar{\mu} \nu}(\zeta)$, with $|\mu|=1,|\alpha|=1$.

Inspired by [9], in this paper, we characterize the k-paranormal, isometric, spectral radius and the numerical radius of the weighted composition operators $C_{\psi, \phi}$ on the Fock space $\mathcal{F}^{p}(\mathbb{C})$, where $\phi(\zeta)=\mu \zeta+\nu,|\mu| \leq 1$ and $\psi(\zeta)=\psi(0) e^{-\mu \bar{\nu} \zeta}$ with $|\mu|=1$.

For a bounded operator $T$ on a normed space $\mathcal{X}$, we have the following definitions

- $T$ is paranormal if $\|T x\|^{2} \leq\left\|T^{2} x\right\|\|x\|$ for every $x \in \mathcal{X}$.

- $T$ is k-paranormal if there exists an integer $k \geq 1$ such that $\|T x\|^{k+1} \leq$ $\left\|T^{k+1} x\right\|\|x\|$ for every $x \in \mathcal{X}$.

- $T$ is normaloid if $\|T\|=r_{\sigma}(T)$, spectral radius of $T$.

- $T$ is spectraloid if $r_{\sigma}(T)=r_{w}(T)$, numerical radius of $T$.

For a bounded linear operator $T$ on a Hilbert space $\mathcal{H}$, we have

- Numerical range is defined as $W(T)=\{\langle T h, h\rangle \mid h \in \mathcal{H},\|h\|=1\}$.

- $T$ is normal if $T^{*} T=T T^{*}$, i.e. $T^{*}$ and $T$ commute.

It is also well known that the following inclusion relationship is true for a bounded operator: Normal $\subset$ Quasinormal $\subset$ Subnormal $\subset$ Hyponormal $\subset$ Paranormal $\subset$ k-Paranormal $\subset$ Normaloid $\subset$ Spectraloid.

\section{Preliminary Results}

In this section, we list the well known results on weighted composition operators on $\mathcal{F}^{p}(\mathbb{C})$.

Theorem 2.1. ([1], Theorem 1) Suppose $\phi: \mathbb{C} \rightarrow \mathbb{C}$ is an analytic function.

(a) If $C_{\phi}$ is bounded on $\mathcal{F}^{2}(\mathbb{C})$, then $\phi(\zeta)=\mu \zeta+\nu$, where $\mu, \nu \in \mathbb{C},|\mu| \leq 1$ and if $|\mu|=1$, then $\nu=0$.

(b) If $C_{\phi}$ is compact on $\mathcal{F}^{2}(\mathbb{C})$, then $\phi(\zeta)=\mu \zeta+\nu,|\mu|<1$.

By ([1], Theorem 2), the converse of the above theorem is also true.

Lemma 2.2. ([7], Proposition 2.1) Let $\psi$ and $\phi$ be two entire functions on $\mathbb{C}$ such that $\psi \not \equiv 0$. Suppose there is a positive constant $K$ such that

$$
|\psi(\zeta)|^{2} e^{|\phi(\zeta)|^{2}-|\zeta|^{2}} \leq K
$$

for all $\zeta \in \mathbb{C}$. Then $\phi(\zeta)=\mu \zeta+\nu$ for $|\mu| \leq 1$. If $|\mu|=1$, then $\psi(\zeta)=\psi(0) e^{-\mu \bar{\nu} \zeta}$.

Theorem 2.3. ([7], Theorem 2.2) Suppose $\psi, \phi$ are analytic functions on $\mathbb{C}$ such that $\psi$ is not identically zero. Then, $C_{\psi, \phi}$ is bounded iff $\psi$ belongs to $\mathcal{F}^{2}(\mathbb{C})$, $\phi(\zeta)=\phi(0)+\lambda \zeta$ with $|\lambda| \leq 1$ and $M(\psi, \phi):=\sup \left\{|\psi|^{2} \exp \left(|\phi(\zeta)|^{2}-|\zeta|^{2}\right) ; \zeta \in\right.$ $\mathbb{C}\}<\infty$.

Proposition 2.4. ([7], Proposition 3.1) Let $\phi(\zeta)=\mu \zeta-\nu,|\mu|=1$ and $\nu$ is an arbitrary complex number. Then $C_{k_{\bar{\mu}}, \phi}$ is an unitary operator on $\mathcal{F}^{2}(\mathbb{C})$. 


\section{Main Results}

In this section, we assume that for analytic functions $\psi, \phi$ on $\mathbb{C}$, there exists a positive constant $M$ such that $|\psi(\zeta)|^{2} e^{|\phi(\zeta)|^{2}-|\zeta|^{2}} \leq M$. Then, by Lemma 2.3, $\phi$ is of the form $\phi(\zeta)=\mu \zeta+\nu,|\mu| \leq 1$. Moreover, in this case when $|\mu|=1$, we have $\psi(\zeta)=\psi(0) e^{-\mu \bar{\nu} \zeta}$.

We begin the proof of our main results by defining the operator $S_{\mu, \nu} f(\zeta):=$ $e^{-\mu \bar{\nu} \zeta-\frac{|\nu|^{2}}{2}} f(\mu \zeta+\nu)$.

Proposition 3.1. For $n \geq 1$, let $\phi(\zeta)=\mu^{n} \zeta+\nu$ and $\psi(\zeta)=\psi(0) e^{-\mu^{n} \bar{\nu} \zeta}$ such that $|\mu|=1$. Then $S_{\mu^{n}, \nu} f(\zeta)=e^{-\mu^{n} \bar{\nu} \zeta-\frac{|\nu|^{2}}{2}} f\left(\mu^{n} \zeta+\nu\right)$ is an isometry on $\mathcal{F}^{p}(\mathbb{C})$.

Proof. For $f \in \mathcal{F}^{p}(\mathbb{C})$, consider

$$
\begin{aligned}
\left\|S_{\mu^{n}, \nu} f\right\| & =\left(\frac{p}{2 \pi} \int_{\mathbb{C}}\left|e^{-\mu^{n} \bar{\nu} \zeta-\frac{|\nu|^{2}}{2}} f\left(\mu^{n} \zeta+\nu\right)\right|^{p} e^{-\frac{p|\zeta|^{2}}{2}} d A(\zeta)\right)^{1 / p} \\
& =\left(\frac{p}{2 \pi} \int_{\mathbb{C}}\left|e^{-\mu^{n} \bar{\nu} \zeta-\frac{|\nu|^{2}}{2}}\right| p\left|f\left(\mu^{n} \zeta+\nu\right)\right|^{p} e^{-\frac{p|\zeta|^{2}}{2}} d A(\zeta)\right)^{1 / p} \\
& =\left(\left.\frac{p}{2 \pi} \int_{\mathbb{C}}\left|e^{-\mu^{n} \bar{\nu} \zeta-\frac{|\nu|^{2}}{2}-\frac{|\zeta|^{2}}{2}}\right| p\left(\mu^{n} \zeta+\nu\right)\right|^{p} d A(\zeta)\right)^{1 / p} .
\end{aligned}
$$

Since $|\mu|=1$, we can derive that $e^{-\mu^{n} \bar{\nu} \zeta-\frac{|\nu|^{2}}{2}-\frac{|\zeta|^{2}}{2}}=e^{\frac{-\left|\mu^{n} \zeta+\nu\right|^{2}}{2}}$. Substituting this in $(3.1)$, we get

$$
\begin{aligned}
\left\|S_{\mu^{n}, \nu} f\right\| & =\left(\frac{p}{2 \pi} \int_{\mathbb{C}}\left|f\left(\mu^{n} \zeta+\nu\right)\right|^{p}\left|e^{\frac{-\left|\mu^{n} \zeta+\nu\right|^{2}}{2}}\right| p d A(\zeta)\right)^{1 / p} \\
& =\left(\frac{p}{2 \pi} \int_{\mathbb{C}}\left|f\left(\mu^{n} \zeta+\nu\right)\right|^{p} e^{\frac{-p\left|\mu^{n} \zeta+\nu\right|^{2}}{2}} d A(\zeta)\right)^{1 / p} \\
& =\|f\| .
\end{aligned}
$$

Thus, $S_{\mu^{n}, \nu}$ is an isometry on $\mathcal{F}^{p}(\mathbb{C})$.

Corollary 3.2. Let $\phi(\zeta)=\mu \zeta+\nu$ and $\psi(\zeta)=\psi(0) e^{-\mu \bar{\nu} \zeta}$ such that $|\mu|=1$. Then $S_{\mu, \nu} f(\zeta)=e^{-\mu \bar{\nu} \zeta-\frac{|\nu|^{2}}{2}} f(\mu \zeta+\nu)$ is a unitary on Hilbert Fock space $\mathcal{F}^{2}(\zeta)$.

Proof. The proof follows from Proposition 2.4 with a change of the sign of $\mu$ in $C_{k_{\bar{\mu} \nu}, \phi}$.

Theorem 3.3. Let $\phi(\zeta)=\mu \zeta+\nu$ and $\psi(\zeta)=\psi(0) e^{-\mu \bar{\nu} \zeta}$ such that $|\mu|=1$. Then, $C_{\psi, \phi}$ is a k-paranormal operator on the Fock space $\mathcal{F}^{p}(\mathbb{C})$. 
Proof. For $f \in \mathcal{F}^{p}(\mathbb{C})$, consider

$$
\begin{aligned}
C_{\psi, \phi}^{k} f(\zeta)= & C_{\psi, \phi} C_{\psi, \phi} \ldots C_{\psi, \phi} f(\zeta) \\
= & \psi(\zeta) \cdot(\psi \circ \phi(\zeta)) \cdot(\psi \circ \phi \circ \phi(\zeta)) \ldots(\psi \circ \phi \ldots \phi(\zeta)) \cdot f(\phi \circ \phi \ldots \phi(\zeta)) \\
= & \psi(\zeta) \cdot \psi(\phi(\zeta)) \cdot \psi\left(\phi^{2}(\zeta)\right) \ldots \psi\left(\phi^{k-1}(\zeta)\right) \cdot f\left(\phi^{k}(\zeta)\right) \\
= & \psi(0) e^{-\mu \bar{\nu} \zeta} \cdot \psi(0) e^{-\mu \bar{\nu} \phi(\zeta)} \cdot \psi(0) e^{-\mu \bar{\nu} \phi^{2}(\zeta)} \ldots \psi(0) e^{-\mu \bar{\nu} \phi^{k-1}(\zeta)} \cdot f\left(\phi^{k}(\zeta)\right. \\
= & \psi(0)^{k} e^{-\bar{\nu} \zeta\left(\mu+\mu^{2}+\mu^{3}+\cdots+\mu^{k}\right)} e^{-|\nu|^{2}\left(\mu^{k-1}+2 \mu^{k-2}+\cdots+(k-1) \mu\right)} \\
& f\left(\mu^{k} \zeta+\nu\left(1+\mu+\mu^{2}+\cdots+\mu^{k-1}\right)\right) .
\end{aligned}
$$

Taking $\rho=\nu\left(1+\mu+\mu^{2}+\cdots+\mu^{k-1}\right)$ along with the fact $\mu^{k} \bar{\rho}=\bar{\nu}\left(\mu+\mu^{2}+\cdots+\mu^{k}\right)$ in $(3.2)$, we get

$$
\begin{aligned}
C_{\psi, \phi}^{k} f(\zeta) & =\psi(0)^{k} e^{-|\nu|^{2}\left(\mu^{k-1}+2 \mu^{k-2}+\cdots+(k-1) \mu\right)} e^{-\mu^{k} \bar{\rho} \zeta} f\left(\mu^{k} \zeta+\rho\right) \\
& =\psi(0)^{k} e^{-|\nu|^{2}\left(\mu^{k-1}+2 \mu^{k-2}+\cdots+(k-1) \mu\right)} e^{-\mu^{k} \bar{\rho} \zeta-\frac{|\rho|^{2}}{2}} e^{\frac{|\rho|^{2}}{2}} f\left(\mu^{k} \zeta+\rho\right) \\
& =\psi(0)^{k} e^{-|\nu|^{2}\left(\mu^{k-1}+2 \mu^{k-2}+\cdots+(k-1) \mu\right)} e^{\frac{|\rho|^{2}}{2}} S_{\mu^{k}, \rho} f(\zeta) .
\end{aligned}
$$

This implies

$$
\begin{aligned}
\left\|C_{\psi, \phi}^{k} f(\zeta)\right\|= & |\psi(0)|^{k}\left|e^{-|\nu|^{2}\left(\mu^{k-1}+2 \mu^{k-2}+\cdots+(k-1) \mu\right)}\left\|e^{\frac{|\rho|^{2}}{2}} \mid\right\| S_{\mu^{k}, \rho} f(\zeta) \|\right. \\
= & |\psi(0)|^{k} e^{\frac{-|\nu|^{2}}{2}\left[\mu^{k-1}+\bar{\mu}^{k-1}+2\left(\mu^{k-2}+\bar{\mu}^{k-2}\right)+\cdots+(k-1)(\mu+\bar{\mu})\right]+\frac{|\rho|^{2}}{2}} \\
& \left\|S_{\mu^{k}, \rho} f(\zeta)\right\|
\end{aligned}
$$

since

$$
\begin{aligned}
|\rho|^{2} & =|\nu|^{2}\left|1+\mu+\mu^{2}+\cdots+\mu^{k-1}\right|^{2} \\
& =|\nu|^{2}\left(1+\mu+\mu^{2}+\cdots+\mu^{k-1}\right)\left(\overline{1+\mu+\mu^{2}+\cdots+\mu^{k-1}}\right) \\
& =|\nu|^{2}\left(1+\mu+\mu^{2}+\cdots+\mu^{k-1}\right)\left(1+\bar{\mu}+\bar{\mu}^{2}+\cdots+\bar{\mu}^{k-1}\right) \\
& =|\nu|^{2}\left[\mu^{k-1}+\bar{\mu}^{k-1}+2\left(\mu^{k-2}+\bar{\mu}^{k-2}\right)+\cdots+(k-1)(\mu+\bar{\mu})+k\right] .
\end{aligned}
$$

Substituting (3.4) in (3.3) and using Proposition 3.1, we have

$$
\left\|C_{\psi, \phi}^{k} f\right\|=|\psi(0)|^{k} e^{\frac{k|\nu|^{2}}{2}}\|f\|
$$


On the other hand,

$$
\begin{aligned}
\left\|C_{\psi, \phi} f\right\| & =\left(\frac{p}{2 \pi} \int_{\mathbb{C}}|\psi(\zeta) \cdot f \circ \phi(\zeta)|^{p} e^{\frac{-p|\zeta|^{2}}{2}} d A(\zeta)\right)^{1 / p} \\
& =\left(\frac{p}{2 \pi} \int_{\mathbb{C}}|\psi(0)|^{p}\left|e^{-\mu \bar{\nu} \zeta}\right|^{p}|f(\mu \zeta+\nu)|^{p} e^{\frac{-p|\zeta|^{2}}{2}} d A(\zeta)\right)^{1 / p} \\
& =\left(\frac{p}{2 \pi} \int_{\mathbb{C}}|\psi(0)|^{p} e^{\frac{p|\nu|^{2}}{2}}\left|e^{-\mu \bar{\nu} \zeta-\frac{|\nu|^{2}}{2}}\right|^{p}|f(\mu \zeta+\nu)|^{p} e^{\frac{-p|\zeta|^{2}}{2}} d A(\zeta)\right)^{1 / p} \\
& =\left(\frac{p}{2 \pi} \int_{\mathbb{C}}|\psi(0)|^{p} e^{\frac{p|\nu|^{2}}{2}}\left|e^{-\mu \bar{\nu} \zeta-\frac{|\nu|^{2}}{2}} f(\mu \zeta+\nu)\right|^{p} e^{\frac{-p|\zeta|^{2}}{2}} d A(\zeta)\right)^{1 / p} \\
& =|\psi(0)| e^{\frac{|\nu|^{2}}{2}}\left(\frac{p}{2 \pi} \int_{\mathbb{C}} \mid S_{\mu, \nu}\left(\left.f(\zeta)\right|^{p} e^{\frac{-p|\zeta|^{2}}{2}} d A(\zeta)\right)^{1 / p}\right. \\
& =|\psi(0)| e^{\frac{|\nu|^{2}}{2}}\left\|S_{\mu, \nu} f\right\| .
\end{aligned}
$$

Applying Proposition 3.1 in (3.6), we get

$$
\left\|C_{\psi, \phi} f\right\|^{k}=|\psi(0)|^{k} e^{\frac{k|\nu|^{2}}{2}}\|f\|^{k} .
$$

From (3.5) and (3.7), we have $\left\|C_{\psi, \phi} f\right\|^{k}=\left\|C_{\psi, \phi}^{k} f\right\|\|f\|^{k-1}$. Hence, $C_{\psi, \phi}$ is a k-paranormal operator on $\mathcal{F}^{p}(\mathbb{C})$.

Theorem 3.4. Let $\phi(\zeta)=\mu \zeta+\nu$ and $\psi(\zeta)=\psi(0) e^{-\mu \bar{\nu} \zeta}$ such that $|\mu|=1$. Then $C_{\psi, \phi}$ is an isometric operator on the Fock space $\mathcal{F}^{p}(\mathbb{C})$ if and only if $|\psi(0)|=$ $e^{\frac{-|\nu|^{2}}{2}}$.

Proof. Assume that $|\psi(0)|=e^{\frac{-|\nu|^{2}}{2}}$. For $f \in \mathcal{F}^{p}(\mathbb{C})$,

$$
\begin{aligned}
\left\|C_{\psi, \phi} f\right\| & =\left(\frac{p}{2 \pi} \int_{\mathbb{C}}|\psi(\zeta) \cdot f \circ \phi(\zeta)|^{p} e^{\frac{-p|\zeta|^{2}}{2}} d A(\zeta)\right)^{1 / p} \\
& =\left(\frac{p}{2 \pi} \int_{\mathbb{C}}|\psi(0)|^{p}\left|e^{-\mu \bar{\nu} \zeta}\right|^{p}|f(\mu \zeta+\nu)|^{p} e^{\frac{-p|\zeta|^{2}}{2}} d A(\zeta)\right)^{1 / p} \\
& =\left(\frac{p}{2 \pi} \int_{\mathbb{C}}|\psi(0)|^{p} e^{\frac{p|\nu|^{2}}{2}}\left|e^{-\mu \bar{\nu} \zeta-\frac{|\nu|^{2}}{2}}\right| p|f(\mu \zeta+\nu)|^{p} e^{\frac{-p|\zeta|^{2}}{2}} d A(\zeta)\right)^{1 / p} \\
& =\left(\frac{p}{2 \pi} \int_{\mathbb{C}}|\psi(0)|^{p} e^{\frac{p|\nu|^{2}}{2}}\left|e^{-\mu \bar{\nu} \zeta-\frac{|\nu|^{2}}{2}} f(\mu \zeta+\nu)\right|^{p} e^{\frac{-p|\zeta|^{2}}{2}} d A(\zeta)\right)^{1 / p} \\
& =|\psi(0)| e^{\frac{|\nu|^{2}}{2}}\left(\frac{p}{2 \pi} \int_{\mathbb{C}} \mid S_{\mu, \nu}\left(\left.f(\zeta)\right|^{p} e^{\frac{-p|\zeta|^{2}}{2}} d A(\zeta)\right)^{1 / p}\right. \\
& =|\psi(0)| e^{\frac{|\nu|^{2}}{2}}\left\|S_{\mu, \nu} f\right\| .
\end{aligned}
$$

Using Corollary 3.2 and $|\psi(0)|=e^{\frac{-|\nu|^{2}}{2}}$ in (3.8), we get

$$
\left\|C_{\psi, \phi} f\right\|=\|f\| \text {. }
$$

Thus, $C_{\psi, \phi}$ is an isometric operator on the Fock space $\mathcal{F}^{p}(\mathbb{C})$.

Next, we derive the spectrum of the weighted composition operators $C_{\psi, \phi}$ on the Fock space $\mathcal{F}^{p}(\mathbb{C})$. 
Theorem 3.5. Let $\phi(\zeta)=\mu \zeta+\nu$ and $\psi(\zeta)=\psi(0) e^{-\mu \bar{\nu} \zeta}$ such that $|\mu|=1$. Then the spectral radius of $C_{\psi, \phi}$ is $|\psi(0)| e^{\frac{|\nu|^{2}}{2}}$.

Proof. By Theorem 3.3, $C_{\psi, \phi}$ is a k-paranormal operator on the Fock space $\mathcal{F}^{p}(\mathbb{C}), p>0$. Since every k-paranormal operator on a normed space is normaloid [6], we have $r_{\sigma}\left(C_{\psi, \phi}\right)=\left\|C_{\psi, \phi}\right\|$. From the proof of Theorem 3.4, we get

$$
\left\|C_{\psi, \phi}\right\|=|\psi(0)| e^{\frac{|\nu|^{2}}{2}}\left\|S_{\mu, \nu}\right\| .
$$

By equation (3.9) and Corollary 3.2, we get $r_{\sigma}\left(C_{\psi, \phi}\right)=\left\|C_{\psi, \phi}\right\|=|\psi(0)| e^{\frac{|\nu|^{2}}{2}}$.

In the following result, we will characterize the spectraloid weighted composition operators on the Hilbert Fock space $\mathcal{F}^{2}(\mathbb{C})$.

Theorem 3.6. Let $\phi(\zeta)=\mu \zeta+\nu$ and $\psi(\zeta)=\psi(0) e^{-\mu \bar{\nu} \zeta}$ such that $|\mu|=1$. Then the numerical radius $C_{\psi, \phi}$ is $|\psi(0)| e^{\frac{|\nu|^{2}}{2}}$.

Proof. For $f \in \mathcal{F}^{2}(\mathbb{C})$ with $\|f\|=1$, we consider

$$
\begin{aligned}
C_{\psi, \phi} f(\zeta)=\psi(\zeta) f(\phi(\zeta)) & =\psi(0) e^{-\mu \bar{\nu} \zeta} f(\mu \zeta+\nu) \\
& =\psi(0) e^{\frac{|\nu|^{2}}{2}} e^{-\mu \bar{\nu} \zeta-\frac{|\nu|^{2}}{2}} f(\mu \zeta+\nu) \\
& =\psi(0) e^{\frac{|\nu|^{2}}{2}} S_{\mu, \nu} f(\zeta)
\end{aligned}
$$

Using (3.10), it follows that

$$
\begin{aligned}
\left\langle C_{\psi, \phi} f, f\right\rangle & =\left\langle\psi(0) e^{\frac{|\nu|^{2}}{2}} S_{\mu, \nu} f, f\right\rangle \\
& =\psi(0) e^{\frac{|\nu|^{2}}{2}}\left\langle S_{\mu, \nu} f, f\right\rangle .
\end{aligned}
$$

By Corolloary 3.2, $S_{\mu, \nu}$ is unitary on the Hilbert Fock space $\mathcal{F}^{2}(\mathbb{C})$. This implies that $S_{\mu, \nu}$ is normal. Hence, by Theorem 1.4-2 of [3], $r_{\sigma}\left(S_{\mu, \nu}\right)=r_{w}\left(S_{\mu, \nu}\right)=$ $\left\|S_{\mu, \nu}\right\|=1$.

Next, we will calculate the numerical radius of $C_{\psi, \phi}$ using (3.11),

$$
\begin{aligned}
W\left(C_{\psi, \phi}\right) & =\left\{\left\langle C_{\psi, \phi} f, f\right\rangle: f \in \mathcal{F}^{2}(\mathbb{C})\right\} \\
& =\left\{\psi(0) e^{\frac{|\nu|^{2}}{2}}\left\langle S_{\mu, \nu} f, f\right\rangle\right\} .
\end{aligned}
$$

From (3.12), we get $r_{w}\left(C_{\psi, \phi}\right)=|\psi(0)| e^{\frac{|\nu|^{2}}{2}} r_{w}\left(S_{\mu, \nu}\right)=|\psi(0)| e^{\frac{|\nu|^{2}}{2}}$.

Corollary 3.7. Let $\phi(\zeta)=\mu \zeta+\nu$ and $\psi(\zeta)=\psi(0) e^{-\mu \bar{\nu} \zeta}$ such that $|\mu|=1$. Then $r_{\sigma}\left(C_{\psi, \phi}\right)=r_{w}\left(C_{\psi, \phi}\right)=\left\|C_{\psi, \phi}\right\|$.

\section{Conclusion}

On the Fock space $\mathcal{F}^{p}(\mathbb{C})$ for $0<p \leq \infty$, where $\psi$ is of the form $\psi(\zeta)=$ $\psi(0) e^{-\mu \bar{\nu} \zeta}, \mu, \nu, \zeta \in \mathbb{C}$, we obtained our main results that show under what conditions the weight composition operator $C_{\psi, \phi}$ is a k-paranormal operator and isometric. Also, we derived the spectral and numerical radius of the weighted composition operator $C_{\psi, \phi}$. 
Acknowledgment. The authors would like to thank the referee for valuable comments and suggestions.

\section{REFERENCES}

[1] B. J. Carswell, B. D. MacCluer and A. Schuster, Composition Operators on the Fock Space, Acta Scientiarum Mathematicarum 69 (2003), 871-887.

[2] C. C. Cowen and B. D. MacCluer, Composition Operators on Spaces of Analytical Functions, Studies in Advanced Mathematics, CRC Press, 1995.

[3] K.E. Gustafon and K. M. Rao, The Numerical Range: The Field of Values of Linear Operators and Matrices, Springer, New York, 1997.

[4] P. V. Hai and L. H. Khoi, Boundedness and compactness of weighted composition operators on Fock Space $\mathcal{F}^{p}(\mathbb{C})$, Acta Mathematica Vietnamica 41 (2016), 531-537.

[5] F. Jafari, B. D. MacCluer, C. C. Cowen and A. D. Porter, Studies on Composition Operators, Contemporary Mathematics 213, American Mathematical Society, 1998.

[6] C.S. Kubrusly, The Elements of Operator Theory, Springer, New York, 2011.

[7] T. Le, Normal and isometric weighted composition operators on the Fock space, Bulletin of the London Mathematical Society 46 (2014), 847-856.

[8] J. H. Shapiro, Composition Operators and Classical Function Theory, Universitext: Tracts in Mathematics, Springer-Verlag, New York, 1993.

[9] L. Zhao, Unitary weighted composition operators on the Fock space of $\mathbb{C}^{n}$, Complex Analysis and Operator Theory 8 (2014), 581-590.

[10] K. Zhu, Analysis on Fock Spaces, Graduate Text in Mathematics, Springer, New York, 2012.

C. Santhoshkumar, Corporate and Industry Relation, Amrita Vishwa Vidyapeetham, Tamilnadu, India 641112 .

e-mail: santhosh_csk@yahoo.com

T. Veluchamy, Dr.SNS Rajalakshmi College of Arts and Science, Saravanampatti, Tamilnadu, India 641035.

e-mail: veluchamy_t@yahoo.com 
